\title{
Exploiting ABCG2 Inhibition to Improve Cancer Therapy
}

Yu Fukuda', Alice Allcock', Juwina Wijaya', Tomoka Gose', Wenwei Lin², Taosheng Chen², John D. Schuetz ${ }^{1}$

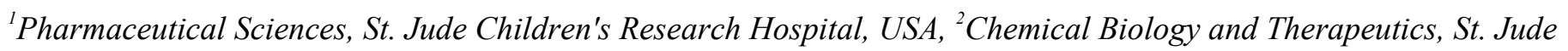
Children's Research Hospital, USA

We have used in vivo murine cancer models to demonstrate the feasibility of ABCG2 inhibition, when coupled with a conventional chemotherapeutic drug, as a strategy to improve therapeutic efficacy for the treatment of both medulloblastoma and acute myeloid leukemia $(1,2)$. We hypothesized that compounds that have the potential to both inhibit ABCG2 and target the pathways activated in cancers would lead to further enhancements in the tumoricidal activity of conventional chemotherapeutics, especially those that are ABCG2 substrates. ABCG2 inhibition was assessed by determining a compound's capability of enhancing the intracellular accumulation of the specific ABCG2 substrate, pheophorbide a. With this robust assay, we performed a high-throughput screen using a library containing $\sim 12,000$ bioactive compounds, including FDA-approved drugs. A number of compounds that were previously unreported as ABCG2 inhibitors were identified. Among these was a kinase inhibitor (KI) that inhibited ABCG2 activity with an IC50< $1 \mu \mathrm{M}$. To determine if this KI directly interacted with ABCG2, a membrane protein cellular thermal shift assay (CETSA) was performed. The CETSA studies demonstrated that our inhibitor stabilized and increased the melting temperature of ABCG2, suggesting direct binding of the KI to ABCG2. By using various combinations of the conventional cancer chemotherapeutic drug, mitoxantrone and the KI, an in vitro screen of murine myeloid leukemic cells revealed synergistic cell killing. Based on such promising preliminary results, these studies will be extended to in vivo studies which, may lead to a therapeutic approach that improves treatment response for cancers expressing ABCG2.

This work was supported by NIH and by the ALSAC.

References:

1) Fukuda $Y$ et al. Upregulated heme biosynthesis, an exploitable vulnerability in MYCN-driven leukemogenesis JCI Insight. 2017;2(15):e92409

2) Morfouace M, Cheepala S, Jackson S, Albert YP, Kawauchi D, Shelat A, Fukuda Y, Sorrentino B, Schuetz JD*, Roussel MF*. (*co-corresponding authors) ABCG2 transporter expression impacts Group3 Medulloblastoma response to chemotherapy. Cancer Res Sep 15;75(18):3879-89, 2015 